\title{
¿Qué ves cuando me ves? Acerca de lo que los programas de proximidad muestran sobre las políticas sociales en el Uruguay.
}

¿What do you see when you see me?: On what the proximity programs show about social policies in Uruguay.

Ximena Baráibar

Docente e investigadora del

De la Facultad de Ciesjo Social

Universidad de la República.

Montevideo, Uruguay. Master

Trabajo Social, Universidad Federal

de Río de Janeiro/Universidad de la

República. Candidata a Doctora po

el Doctorado en Ciencias Sociales

la Facultad de Ciencias Sociales de la

Universidad de la República.

Fecha de recepción:

1.3.17

Fecha de aceptación

21.4.17

\section{Resumen}

En el 2005, asume el gobierno nacional en el

Uruguay una fuerza política de izquierda (Frente

Amplio). En un contexto marcado por las conse-

cuencias de la crisis del 2002, desarrolló como una

de sus líneas fundamentales de actuación la asis-

tencia a la pobreza. Esto se expresa en la creación

del Ministerio de Desarrollo Social y la implementación de variados programas sociales. A partir de 2012, el segundo gobierno del Frente Amplio, desarrolla programas de proximidad: Uruguay Crece Contigo, Jóvenes en Red y Cercanías. Estos son presentados como innovadores en tanto desarrollan trabajo de proximidad con la población destinataria y son de carácter interinstitucional. Este trabajo pone foco en la relación entre estos programas y las restantes prestaciones del Estado, considerando el diseño y la implementación. Interesa problematizar qué es lo que la existencia de estos programas permite visualizar sobre el funcionamiento de las políticas sociales en el Uruguay actual. Se concluye que la relación entre los programas de proximidad y las restantes prestaciones del Estado es ausente por momentos, compleja en otros y con riesgos de 
desresponsabilización de parte de las prestaciones públicas no focalizadas. La existencia de los programas de proximidad constituye una nueva expresión de la desigualdad en el acceso a las políticas públicas y más ampliamente, otra muestra de la desigualdad social persistente.

Palabras claves: políticas sociales, programas de proximidad, desigualdad, Uruguay

\section{Abstract}

In 2005, the national government in Uruguay was assumed by a leftist political force (Frente Amplio). In a context marked by the consequences of the crisis of 2002, it developed as one of its fundamental lines of action the assistance to poverty. This is expressed in the creation of the Ministry of Social Development and a varied set of social programs. The second government of Frente Amplio, develops - from 2012 - proximity programs: Uruguay Crece Contigo, Jóvenes en Red y Cercanías. These are presented as innovators, basically in two aspects: insofar as they develop a proximity approach to the target population and are of an interinstitutional nature. Based on a study carried out on Proximity Programs, these paper focuses on the relationship between them programs and the remaining state benefits, considering their design and implementation. It is interested in problematizing what the existence of these programs allows to visualize on the operation of the social policies in current Uruguay. It is concluded that the relationship between the proximity programs and the other state benefits is absent at times, complex in others and with risks of disengagement from public non-targeted benefits. The existence of the proximity programs is a new expression of inequality in access to public policies and, more broadly, another sign of persistent social inequality.

Key words: social policies, proximity programs, inequality, Uruguay

\section{Introducción}

Desde fines del siglo XX, América Latina asiste a un proceso de ampliación, diversificación e institucionalización de la política asistencial. Distintos programas sociales dirigidos específicamente a la pobreza y el desarrollo de institucionalidades especializadas en su gestión, son parte de la expresión de esta realidad. Dentro de la política asistencial, los programas de transferencias condicionadas, adquieren centralidad. En 18 países de América Latina y el Caribe y beneficiando al 19\% de su población, estos programas se proponen objetivos de corto y largo plazo, en tanto, apuntan a reducir el impacto de la escasez de ingresos en tiempos inmediatos y también la pobreza en el largo plazo, a partir de acrecentar el capital humano (Cecchini y Madariaga, 2011).

Avanzado el siglo XXI a lo anterior se suman programas de proximidad basados en la idea de que no alcanza con exigir condiciones de participación para asegurar que los beneficios monetarios incentiven el consumo de servicios. Sus promotores entienden que es preciso revisar los modos de intervención con que las políticas llegan a sus beneficiarios y considerar los modelos de acompañamiento como estrategia para la atención de poblaciones vulnerables de manera de suplementar ciertos servicios para llegar a poblaciones que por alguna razón, no tienen cobertura (Jara y Sorio 2013: 3,19).

Este camino es el que ha seguido el Uruguay desde el 2005 cuando el Frente Amplio accede al gobierno nacional, creando el Ministerio de Desarrollo Social (MIDES), el Gabinete Social y el Consejo Nacional de Políticas Sociales, como instancias de coordinación de políticas sociales.También desarrolla tres marcos a partir de los cuales se implementa un conjunto variado de programas en diversas áreas de la política social. Entre 2005 y 2007, desarrolla el Plan Nacional de Atención a la Emergencia Social (PANES).A partir del 2008, se pone en marcha el Plan de Equidad y aún permaneciendo este, en 2011 es aprobada la Reforma Social.

Es muy variada la traducción de esos marcos en términos de programa, pero adquieren centralidad -en población atendida y recursos destinados a los mismos- las transferencias condicionadas de ingreso (Ingreso Ciudadano y Asignaciones Familiares). A partir del 2012 -dentro del segundo gobierno frenteamplista- y en un contexto económico y social distinto al del 2005, se desarrollan los programas de proximidad: Cercanías, Jóvenes en Red y Uruguay Crece Contigo.

Más allá de ciertas diferencias, estos programas presentan elementos comunes: son altamente focalizados, definen un abordaje por un tiempo delimitado y territorial. Poseen baja materialidad e institucionalidad, sofisticados sistemas de registro y monitoreo y se proponen vincular o revincular a los destinatarios con el mundo formal del trabajo y los servicios públicos. Destacan como innovaciones el trabajo de proximidad con la población destinataria y la interinstitucionalidad de las propuestas. 
Estos programas se implementan en un contexto de aumento del gasto social expresado en el mayor desarrollo de políticas sociales y en una mejora en varios indicadores sociales (indigencia, pobreza, desempleo).

Este trabajo tiene como objetivo problematizar lo que la existencia de los programas de proximidad indica sobre el resto de la política pública (fundamentalmente, la política social) y en los impactos que en este sentido puedan estar generando los nuevos programas. Para esto, toma como base una investigación desarrollada durante 2016: "Estudio de los dispositivos de proximidad en las estrategias de intervención de los programas Cercanías, Jóvenes en Red y Uruguay Crece Contigo del Ministerio de Desarrollo Social"; ${ }^{1}$ se considera y analiza tanto el diseño de los programas como la implementación de los mismos a partir del relato realizado por quienes allí se desempeñan.

Luego de esta introducción, el trabajo se organiza en cuatro partes. En la primera se conceptualiza la política social y específicamente la asistencial y se presentan brevemente sus expresiones históricas. Luego se describen los elementos que han configurado el campo asistencia en el Uruguay a partir del 2005. La tercera parte se centra en los programas de proximidad, especialmente en su relación con el mundo formal de trabajo y el conjunto de instituciones a las que aspiran a vincular. El trabajo se cierra con un punto de síntesis y conclusiones.

\section{Política social y política asistencial}

Entiende Grassi $(2008: 30,47)$ que la política social expresa el cómo y hasta donde una sociedad asegura la reproducción de sus miembros y por tanto, la forma en que resuelve su propia reproducción. Por ella se expresa y se produce un modo de realización de la reproducción que supone criterios de mayor o menor socialización de la satisfacción de las necesidades y mayor o menor cantidad y calidad de los satisfactores.

Además, estas cuantías indican y producen distinciones sociales y por tanto, por ellas se contribuye a constituir sujetos provistos de diferentes atributos. Para la autora, las políticas sociales conllevan un concepto de necesidad implícito, del que depende la determinación de aquellas a cuya satisfacción se orientarán las intervenciones en política social y a quienes le son reconocidas. De esta manera, el Estado delimita los ámbitos de responsabilidad de atención de los problemas sociales, establece los alcances de su intervención y define a los sujetos que justifican tales intervenciones. Así, los alcances, sentidos e intereses de la política social se disputan y expresan en la definición de quien es y como se define como merecedor de atención, el tipo de atención a recibir y sus condiciones y obligaciones (Grassi, 2008: 31,47).

Lo señalado implica que la política social no encuentra un sujeto pre - constituido, sino que en la identificación del destinatario y en el encuentro con quienes se nombra, le da existencia. Aún cuando los sujetos pre existen a la política social, esta participa del poder de constitución de sujetos, sean como asistidos, pobres, ciudadanos, trabajadores (Grassi, 2013: 281).

Soldano y Andrenacci (2005: 32) entienden que las políticas sociales constituyen: (...) aquellas intervenciones públicas que regulan las formas en que la población se reproduce y socializa (sobrevive fisicamente y se inserta en el mundo del trabajo y en el espacio sociocultural del Estadonación) y que protegen a la población de situaciones que ponen en riesgo esos procesos".

De acuerdo a los autores, esas intervenciones se expresan en tres campos de actuación: políticas de trabajo, políticas de servicios universales y política asistencial. La responsabilidad de esas áreas es dependiente de clivajes temporales y espaciales, de las historias y estructuras de cada país. Soldano y Andrenacci (2005) identifican tres clivajes temporales: el Estado capitalista clásico, el Estado social y el Estado capitalista neoclásico. A los efectos de este trabajo, serán presentados los contenidos de la segunda y tercera de estas expresiones.

Los elementos característicos del Estado social aparecen en Europa Occidental durante las últimas décadas del siglo XIX y se transforman en rasgos dominantes luego de la crisis financiera de 1929-30, consolidándose después de la Segunda Guerra Mundial.

La generalización del empleo asalariado convirtió a las relaciones salariales en el modo predominante de vida de los sectores no poseedores de capital. A través de la política de trabajo: “(...) la política social transformó este modo de participación en la división social del trabajo en un status garante de condiciones de vida, asegurando contra las irregularidades del ciclo económico y los riesgos individuales de la vida activa, capaz incluso de habilitar procesos de movilidad ascendente" (Soldano y Andrenacci, 2005: 36).

Además, el Estado intervino regulando las formas de los contratos de trabajo. La consolidación del status de los asalariados, implicó también la generalización de un sistema de seguros basado en la protección socializada de los riesgos de la vida activa, tales como desempleo, accidentes de trabajo, enfermedad, vejez y muerte (Soldano y Andrenacci, 2005: 36).

La política de servicios universales se traduce en la expansión universalizante del sistema de educación, en la creación de una red de salud pública y en servicios de infraestructura social básica subsidiados o gestionados directamente por el Estado (Soldano y Andrenacci, 2005: 60).

Aunque la política social a través del trabajo y políticas universalizantes supusieron la gradual desaparición de políticas sobre grupos de riesgo, la política asistencial siguió constituyendo una tercera vertiente de la acción pública y se pone de manifiesto ante sectores sociales que no pueden proveerse ingresos por el mercado de trabajo y que, 
por diferentes razones, a pesar de existir provisión pública de servicios, no acceden a ella. Así, la política asistencial se expresó en programas sociales dirigidos a individuos en situaciones de pobreza hasta su absorción o reabsorción en el mercado de trabajo (Andrenacci, 2009: 13).

A partir de los años 90, ubican Soldano y Andrenacci (2005: 69) al Estado capitalista neoclásico, el que supuso una profunda reforma de la política social. La política de trabajo contribuye a reformar el mercado garantista, orientándose las intervenciones públicas a la desregulación del mercado de trabajo y la reducción del peso de los costos laborales. Esto fue expresado en la flexibilización de las pautas contractuales del fordismo y en la remercantilización de la cobertura de los riesgos de la vida activa a través de los cuasimercados de gestión privada.

El enfoque neoliberal también tuvo impactos en lo que hace a la política de servicios universales, implicando la revisión de las competencias públicas. En educación y salud, las estrategias se orientaron a la desconcentración y descentralización, sumando en la infraestructura social, la privatización de servicios públicos (Soldano y Andrenacci (2005: 69-70).

Finalmente, en este período se amplifica la política asistencial, expresada en una red mínima de intervenciones sobre los grupos percibidos como portadores de mayor riesgo. Se multiplicaron los programas sociales, con objetivos, plazos y financiamientos limitados (Soldano y Andrenacci (2005: 70).

Filgueira $(2015: 50,73)$ comparte la identificación de etapas del Estado realizada por los autores presentados y destaca la existencia de un cuarto momento del Estado social, que se configura como un modelo abierto y de orientación exportadora, pero con participación renovada del Estado. Destaca algunas esferas de la política social:jubilaciones, pensiones y otras transferencias monetarias a los adultos mayores y a familias con hijos. Acceso a servicios y aseguramiento de salud y cambios en la oferta y rangos de edad cubiertos por la educación. Finalmente indica la protección al trabajador, a través de seguro de enfermedad, desempleo, sumado a políticas de derechos laborales.

Lo señalado en último lugar implica una combinación particular de las expresiones de la política social señaladas. El Estado retoma su actuación en la política de trabajo y en los servicios universales, pero a diferencia del período posterior a la Segunda Guerra Mundial mantiene y expande la política asistencial.

De acuerdo a Filgueira (2015: 77) en materia asistencial, la evolución más importante ha sido la construcción o expansión de sistemas de transferencias monetarias no contributivas a las familias con hijos, siendo el vehículo dominante el de los programas de transferencias condicionadas (PTC).
Surgidos en Brasil a mediados de la década de 1990 y con mayor desarrollo a partir de la mitad de la misma con el inicio en México del Programa de Educación, Salud y Alimentación (PROGRESA), estos programas se han consolidado como instrumentos privilegiados de la política contra la pobreza (Cecchini y Madariaga, 2011:9).

Cecchini y Martínez (2011: 100) señalan que los PTC difieren en las prestaciones que ofrecen y la modalidad con la que se entregan, los niveles geográficos donde funcionan, la proporción de población atendida, sus vinculaciones interinstitucionales y el nivel de inversión comprometido. Aún así, identifican una estructura común en esta línea de actuación pública.

Se trata de programas de subsidio a la demanda por medio de transferencias monetarias directas focalizados en la población pobre y/o extremadamente pobre, que tienen como objetivo apoyar a las familias para que puedan salir de la pobreza y superar una situación de crisis de ingreso en el corto plazo. Los programas utilizan principalmente transferencias monetarias de libre uso (y no de uso predeterminado) ya que se considera que los hogares son los que mejor uso pueden hacer de dichas transferencias sobre la base de sus preferencias (Cecchini y Madariaga, 2011)

A los objetivos mencionados, se suman otros orientados a disminuir la incidencia de la pobreza en el largo plazo a partir de acrecentar el capital humano. Esos objetivos se expresan en la condicionalidad del beneficio, la que toma la forma de exigencia de asistencia al sistema educativo y de salud (Cecchini y Madariaga, 2011: 9).

La condicionalidad es también justificada en el quiebre de la lógica anterior de actuación del Estado (donde se traspasaban recursos a beneficiarios que los recibían en forma pasiva) y se la entiende como promotora de la corresponsabilidad, permitiendo a los beneficiarios mejorar sus condiciones de vida y salir de los programas en el menor tiempo posible.

Avanzado el siglo XXI, a los PTC se suman las políticas de acompañamiento familiar. Jara y Sorio (2013:2) señalan que a pesar de los logros de las transferencias condicionadas, estas enfrentan desafios para mejorar su capacidad de generar capital humano en las familias más pobres y definir itinerarios para salir de la pobreza. Uno de los retos más importantes es ampliar el alcance de sus resultados, en tanto estos programas aumentan la utilización de servicios, pero su impacto final es menos convincente.

Dentro de los factores que explican la limitación de los resultados, Jara y Sorio (2013: 2, 19) señalan la baja calidad de los servicios y la restringida capacidad del dinero asociado al cumplimiento de condicionalidades para lograr cambios de comportamiento sostenibles. Asimismo indican que no alcanza con exigir condiciones de participación en los programas para asegurar que los beneficios monetarios incentiven el consumo de otros 
servicios, siendo necesaria una alternativa que permita abordar las situaciones familiares donde es menos probable que los incentivos actúen por sí solos.

También destacan las autoras que existe acuerdo entre diferentes responsables de política en que los modelos más tradicionales de servicios de corte sectorial, parecen no producir los efectos esperados. Como consecuencia, crece el interés por programas que apuestan a intervenciones intersectoriales que persiguen resultados integrales (Jara y Sorio, 2013:3, 9).

Asimismo entienden que es preciso revisar los modos de intervención con que las políticas llegan a sus beneficiarios y considerar los modelos de acompañamiento como estrategia para la atención de poblaciones vulnerables de manera de suplementar ciertos servicios para llegar a poblaciones prioritarias que por alguna razón, no tienen cobertura (Jara y Sorio, 2013: 3,12)

El acompañamiento familiar se configura como:

“(...) un tipo de consejería, altamente estructurada, que involucra procesos de largo plazo y se orienta a metas vinculadas a modificar prácticas para mejorar la seguridad $y$ el bienestar de las personas. Su tecnología principal es la presencia de un profesional o educador especialmente entrenado para brindar apoyo psicosocial familiar o personal. La intervención se apoya en un riguroso sistema de registro que permite dar seguimiento a procesos y evaluar logros" (Jara y Sorio, 2013: 3).

De acuerdo a Jara y Sorio (2013: 3), los objetivos del acompañamiento familiar son diversos, aunque es posible identificar algunos genéricos como promover la autonomía familiar, desarrollar capacidades para la autogestión y mejorar la participación en la estructura de oportunidades. Otros objetivos ponen foco en temas de cada ámbito de intervención: apoyo familiar para alcanzar condiciones que faciliten procesos de salida de la pobreza; consejería para la formación de competencias parentales que permitan el buen cuidado y crianza de los niños; acompañamiento en procesos de inserción y permanencia en actividades económicas o laborales.

Indican las autoras que aunque se sabe poco de los efectivos resultados de estos programas, son diversas las ventajas para las políticas sociales: favorecen la transformación de comportamientos y prácticas de las familias, dan seguimiento a la trayectoria de los integrantes que se encuentran en procesos de sustitución de comportamientos de riesgo y permiten organizar mejor en beneficio de la familia los recursos que entregan otros programas y servicios (Jara y Sorio, 2013: 4)

\section{La política asistencial en el Uruguay de los últimos años}

En marzo de 2005 y por primera vez en la historia del Uruguay, asume un gobierno a cargo de la izquierda política (Frente Amplio) en un contexto marcado por elementos negativos, luego de la crisis del año 2002. La población en situación de pobreza superaba los guarismos históricos y alcanzaba en el año 2004 al 39,9\% de la población, teniendo la indigencia una incidencia del 4,7\% (MIDES y OPP, 2013). También fueron visualizables los impactos en el mercado de trabajo. En el año 2000 la tasa de desempleo se ubicaba en el 13,6\% llegando al 17\% en el año 2004. Por su parte, en el 2004 el valor del índice de Gini era de 0.471 para el total del país (MIDES y OPP, 2013).

El Frente Amplio otorgó jerarquía a la agenda social y desarrolló como una de sus líneas principales de acción la asistencia a la pobreza. Esto puede ser visualizado en las innovaciones institucionales, expresadas fundamentalmente en la creación el Ministerio de Desarrollo Social (MIDES) y en el amplio y diversificado conjuntos de programas desarrollados ubicados en tres marcos: el Plan de Atención Nacional a la Emergencia Social (PANES) implementado entre 2005 y 2007; el Plan de Equidad iniciado en 2008 y con vigencia hasta la actualidad y la Reforma Social, aprobada en el año 2011 y que también se mantiene vigente al día de hoy

El nuevo gobierno, mediante la ley 17.866 crea el MIDES, estableciendo trece competencias agrupables en cuatro esferas. Le compete implementar, ejecutar y coordinar la puesta en marcha del Plan de Atención Nacional a la Emergencia Social. La segunda esfera refiere a la gestión de políticas y planes en las áreas de juventud, familia, adultos mayores, personas en situación de discapacidad y desarrollo social en general. La tercer esfera tiene que ver con la articulación y coordinación de acciones, planes y programas intersectoriales y finalmente, las competencias del MIDES también están vinculadas a la organización y gestión de un sistema de información de la oferta pública social.

En mayo de 2005, mediante la Ley 17.869 se crea el Plan Nacional de Atención a la Emergencia Social, pensado como respuesta a la emergencia social, la que es entendida como consecuencia de las dificultades de inserción social y comprobada por los indicadores de pobreza e indigencia.

Luego la ley define los programas integrantes del PANES: Plan Alimentario Nacional y los Programas de Emergencia Sanitaria; de Ingreso Ciudadano; de Educación en Contextos Críticos; de Empleo Transitorio; de Asentamientos Precarios y Pensiones y Programa de Alojamiento a las Personas en Situación de Calle.

El PANES buscó proporcionar asistencia contingente fundamentalmente a través de transferencias monetarias y alimentarias. Pero también persiguió una finalidad de más largo plazo, tendiente a la reinserción de los beneficiarios. Se esperaba que estos alcan- 
zaran instrumentos que les permitieran salir de la pobreza en el mediano plazo. En este sentido se orientaron las intervenciones en capacitación, educación y alfabetización, junto a experiencias de participación social y laboral (Amarante et al, 2009: 4).

El Ingreso Ciudadano consistió en una transferencia de dinero sujeta al cumplimiento de contrapartidas, básicamente referidas a educación y salud. Se trató de un monto fijo de dinero y focalizado en hogares con ingresos extremadamente bajos y con carencias críticas en sus condiciones de vida (Ley 17.869).

Cumplidos los dos años de ejecución del PANES, el gobierno diseñó el Plan de Equidad (PE) que comenzó a implementarse en enero de 2008 y abarca a los beneficiarios del PANES, pero su población objetivo es el conjunto de los ciudadanos, en especial quienes se encuentran en situación de vulnerabilidad social (Consejo Nacional de Políticas Sociales, 2008: 18)

Constituye una línea de acción de largo plazo que se propone modificar desigualdades económicas, de género, étnicas, etarias, entre otras, para lo cual se organiza en dos grandes conjuntos de acciones. Los componentes estructurales de la matriz de protección social refieren a procesos generales que reforman total o parcialmente las políticas públicas considerada relevantes en materia de equidad: reforma tributaria, de salud y educativa y el plan de igualdad de oportunidades y derechos. (Consejo Nacional de Políticas Sociales, 2008:31).

El otro conjunto de acciones se enmarca en la Red Moderna de Asistencia e Integración Social (RAIS), destinada particularmente a aquellos sectores socioeconómicos que tienen restringidas sus oportunidades de incorporarse al mercado de empleo. Se señala que esta Red no se traduce en simples programas de ayuda a los pobres, sino que pretende articularse con el sistema de seguridad social y las políticas universales que garantizan los derechos sociales (Consejo Nacional de Políticas Sociales, 2008: 9, 30).

Forman parte de la RAIS las prestaciones sociales no contributivas (asignaciones familiares y pensiones a la vejez), seguridad alimentaria, trabajo protegido, promoción de cooperativas sociales y emprendimientos productivos, políticas de educación para la infancia y la adolescencia, políticas de promoción e inclusión social y orientadas a fomentar la participación social (Consejo Nacional de Políticas Sociales, 2008: 8).

En el año 2010, asume el gobierno nuevamente el Frente Amplio, en un contexto distinto al del año 2005. En el 2011, la incidencia de la pobreza se ubicaba en el 14.3\% y la indigencia en el $0.5 \%$. La tasa de desempleo correspondía al $6.3 \%$ en el total del país urbano y el valor del índice de Gini para el total del país era de 0.403. Por su parte, el gasto público social medido en pesos constantes continuó la tendencia ininterrumpida iniciada en 2004. Tomando como referencia ese año, el crecimiento fue de 75\% del total (MIDES et al, 2013).
Pese a ese contexto, la política asistencial continuó ampliándose y diversificándose. En el año 2011 el Consejo Nacional de Políticas Sociales, aprueba la Reforma Social que se propone avanzar en la nueva matriz de protección social: en sus componentes estructurales, en los asistenciales y en los nexos entre ambos. Se entiende que solamente de esta forma se podrá reducir sustentablemente la pobreza, así como la segmentación social y la desigualdad (Consejo Nacional de Políticas Sociales, 2011: 11).

El documento señala que es necesario profundizar las políticas desarrolladas, generar nuevas y fortalecer la llegada de todas las políticas a los sectores más vulnerables. También se hace referencia a la segmentación socio territorial y la necesidad de articular las dimensiones sectoriales con las territoriales (Consejo Nacional de Políticas Sociales, 2011:16,18).

La Reforma Social define tres componentes de la matriz de protección social: aquellos dirigidos al conjunto de los habitantes del país; los orientados al $20 \%$ de la población en situación de pobreza y vulnerable a la pobreza y los destinados al 3\% de la población en situación de indigencia y vulnerable a la indigencia (Consejo Nacional de Políticas Sociales, 2011:20).

En este marco, en el 2012 surgen los programas de proximidad (originalmente denominados programas prioritarios): Cercanías, Jóvenes en Red y Uruguay Crece Contigo.

\section{Los Programas de Proximidad}

La Estrategia Nacional de Fortalecimiento de las Capacidades Familiares, Cercanías tiene como objetivo general mejorar la eficiencia de las intervenciones del Estado ante situaciones de vulneración de derechos, considerando a la familia como sujeto de intervención (MIDES, 2016: 7). Supone un esfuerzo interinstitucional para la atención de familias en situación de extrema vulnerabilidad y basa su accionar en la articulación institucional con otros programas, servicios e instituciones existentes en los territorios para el efectivo acceso a prestaciones, derechos y servicios. Promueve un cambio de gestión en las instituciones para superar fragmentaciones y superposiciones; mejorar la articulación de los servicios a nivel territorial, el trabajo en red y la integralidad en el primer nivel de atención. También se propone desarrollar un trabajo de proximidad con las familias que presenten mayor índice de carencias críticas y de vulnerabilidad socio-familiar para garantizar un acceso rápido y eficiente a las prestaciones sociales básicas existentes (MIDES et al, 2012b:2).

La implementación del trabajo se organiza a partir de una Comisión Político Técnico Interinstitucional, una Unidad de Gestión, Espacios Locales de articulación de servicios y Equipos Territoriales de Atención Familiar (ETAF), los que desarrollan la metodología de proximidad para el acompañamiento a las familias (MIDES et al, 2012b:3). 
Son variadas las funciones definidas para los ETAF, entre las que interesa destacar: la atención a las familias en situación de extrema vulneración actuando sobre las diferentes problemáticas que las atraviesan para el desarrollo de sus integrantes. Cuando corresponda, deben coordinar con equipos e instituciones de la zona para dar una respuesta articulada. Forma parte de sus tareas la realización de un diagnóstico que incorpore la perspectiva de la familia sobre los derechos vulnerados, así como dificultades y capacidades que se presentan para su restitución. Tendrán que acordar un plan de acción con la familia y/o con las instituciones derivantes y apoyar el fortalecimiento del grupo familiar, promoviendo los cambios vinculares, de organización y sostén necesarios para el bienestar de sus integrantes. Deberán facilitar a la familia el acceso a los derechos sociales, bienes y servicios sociales, económicos y culturales. También tienen que identificar y promover la activación de las redes familiares y comunitarias de sostén del grupo familiar y trabajar el egreso con la familia y con el equipo encargado de su seguimiento (de INAU ${ }^{2}$ o MIDES). Finalmente es parte de su tarea el registro adecuado y actualizado del trabajo con las familias en los sistemas informáticos (MIDES, 2016: 9 - 10).

Jóvenes en Red, está dirigido a adolescentes y jóvenes de 14 a 24 años, que no estudian ni culminaron el ciclo básico, pertenecen a hogares con ingresos por debajo de la línea de pobreza y no tienen un empleo formal. El documento fundacional indica que la situación de estos adolescentes y jóvenes no se debe principalmente a temas vinculados a la voluntad de los mismos, sino a la existencia de fenómenos estructurales que condicionan fuertemente la concreción de aspiraciones de desarrollo educacional y laboral (MIDES et al, 2012 a: 8, 11)

También señala el documento que las propuestas para adolescentes y jóvenes tienen una cobertura relativamente menor a las de la niñez, pero que sin embargo, existe en todo el territorio nacional una amplia gama de propuestas educativas, socio educativas y de protección social que vinculan a los jóvenes con las instituciones educativas y del mercado laboral (MIDES et al, 2012 a: 5).

Este programa se origina en una valoración de los ámbitos educativos y laborales como factores claves en el desarrollo de las capacidades y logros de autonomía. La documentación también destaca que uno de los desafios más importantes para Uruguay implica brindar respuestas eficaces a colectivos juveniles que presentan altos niveles de exclusión del mercado laboral y el sistema educativo. Para obtener los resultados esperados, los programas deben ser integrales lo que lleva a elaborar una propuesta de carácter interinstitucional (MIDES et al, 2012 a: 7-8).

Jóvenes en Red tiene como objetivo general promover el ejercicio de derechos de los adolescentes y jóvenes de 14 a 24 años, desvinculados del sistema educativo y del mercado formal de empleo desde un abordaje integral, territorial y en comunidad (MIDES et al, 2012 a: 8).
El programa define cuatro objetivos específicos, comenzando por identificar demandas e intereses de la población objetivo y diseñar una propuesta adecuada. También debe generar oportunidades sustentables de inserción laboral y/o educativa, con énfasis en la educación formal y brindar acreditación y/o certificación de los logros adquiridos tanto en lo educativo formal como en las competencias específicas. Finalmente debe promover el acceso de los destinatarios a la Red de Asistencia e Integración Social (MIDES et al, 2012 a: 8).

El Programa se desarrolla en base a cuatro componentes. El primero está referido a la convocatoria, apoyo, acompañamiento a los jóvenes y al seguimiento para el egreso. El segundo componente se vincula a los espacios de formación y el tercero está centrado en aspectos laborales. Finalmente, hacen parte del cuarto componente la proyección y fortalecimiento comunitario.

La estructura operativa se conforma por el Equipo de Gestión orientado a establecer una dinámica que posibilite el cumplimiento de los objetivos y el Equipo de Trabajo Territorial, con la tarea de desarrollar los cuatro componentes mencionados (MIDES et al, 2012 a: 18).

Los Términos de Referencia para el educador/a del equipo socio educativo territorial, definen las actividades que tendrá a su cargo: debe elaborar un mapa local geo-referenciado para ubicar a la población objetivo, los servicios educativos, laborales y juveniles existentes en la comunidad o eventualmente su ausencia.También debe implementar el espacio socioeducativo territorial y referenciar educativa y socialmente al grupo de jóvenes participantes. Tiene a su cargo la implementación de los componentes I y II del programa y articular los componentes III y IV con los actores de la educación formal, no formal, sociedad civil, entre otros. Es parte de su tarea realizar la planificación educativa y social a nivel local e implementar un acuerdo educativo que permita establecer los compromisos con cada participante del programa (joven, referentes educadores, familia, instituciones, etc). También deberá diseñar y acompañar estrategias de trabajo con las familias referentes de los y las jóvenes con el fin de fortalecer el vínculo y los procesos educativo-laborales de los participantes. También implementará el dispositivo de acompañamiento y seguimiento a las inserciones educativas y laborales de los jóvenes. Deberá promover y participar de los espacios colectivos a nivel territorial en las temáticas de educación, trabajo, participación y jóvenes. Es parte de su tarea articular, coordinar y complementar las estrategias educativas, laborales y sociales de los y las jóvenes con las propuestas de talleres temáticos específicos en función de las demandas y necesidades de los y las jóvenes, su entorno y sus familias. Finalmente deberá acompañar el proceso de sistematización de lo realizado en los equipos socioeducativos; sistematizar las demandas educativas no satisfechas y conocer y transmitir la información asociada a los jóvenes, necesaria para la evaluación y monitoreo (MIDES, s/f: 3 - 4).

Finalmente, Uruguay Crece Contigo se propone como objetivo general consolidar un sistema de protección integral a la primera infancia a través de una política pública 
que garantice los cuidados y protección adecuados de las mujeres embarazadas y el desarrollo de niños y niñas menores de 4 años, para un desarrollo integral, desde una perspectiva de derechos (UCC, s/f: 2).

El programa define cuatro objetivos específicos. En primer lugar generar conocimiento para el diseño, monitoreo y evaluación de las políticas de protección dirigidas a la primera infancia a través de la articulación interinstitucional de la información existente y la generación de información necesaria. También se orienta a desarrollar acciones educativas y de promoción para incidir en el comportamiento y la calidad de vida de las familias y mejorar la calidad de los servicios, para favorecer un desarrollo infantil apropiado. También se propone disminuir la incidencia de los factores de riesgo socio sanitarios y el daño en niños y niñas menores de 4 años y de mujeres embarazadas, priorizando las situaciones de mayor vulnerabilidad y finalmente fortalecer las instituciones, redes y dispositivos territoriales para garantizar el mejor desarrollo del sistema de protección social vinculado a la primera infancia (UCC, s/f: 2 -3).

La documentación parte de considerar la relevancia de la primera infancia, entendiendo que es en este período que se sientan las bases de todo el desarrollo posterior del individuo. Se señala que las neurociencias demuestran la trascendencia de atender la niñez desde la gestación, y durante los primeros años de vida. La intervención también se justifica en tanto mecanismo que facilita la reducción de la pobreza y la desigualdad y como una cuestión de derechos, específicamente a un buen comienzo en la vida (UCC, s/f: 8-9).

El diseño y la ejecución del programa se definen de carácter interinstitucional e intersectorial, garantizando la articulación gubernamental necesaria y la sustentabilidad de las acciones. Específicamente, UCC es entendido como espacio de articulación y fortalecimiento del trabajo que viene desarrollando el Estado desde diferentes instituciones (UCC, s/f: 1).

La estructura organizativa del programa está conformada por una Mesa de Coordinación Interinstitucional responsable de la gestión política de UCC. La Unidad Técnica Central es quien debe fijar los objetivos de trabajo de acuerdo a la planificación estratégica, coordinar con las sectoriales y realizar el seguimiento y evaluación de las acciones. También forman parte de la estructura organizativa, los equipos de cercanía integrados por facilitadores, supervisores y operadores. Estos tienen como tarea principal el realizar el acompañamiento socio-sanitario a las familias (OPP/UCC, 2014 a: 29-31).

Los términos de referencia para la contratación de operadores, indican que son sus funciones: planificar la intervención con las familias para las cuales será referente, habiendo identificado mapa de actores, servicios y redes locales. Deberá atender las situaciones críticas identificadas y dar seguimiento según criterios acordados y protocolos de intervención. También hace parte de sus tareas el promover cambios vinculares, organiza- cionales y de sostén en las familias, que contribuyan con el bienestar y el adecuado desarrollo infantil y deberá facilitar el acceso de las familias a la malla de protección social de todas aquellas prestaciones a las cuales tiene derecho y que por diferentes motivos no accede. También deberá coordinar sus acciones con la red de protección social y las instituciones que forman parte de la malla de protección, pertenecientes a su territorio de referencia. Entre las funciones también se encuentra el aportar información a la supervisión de todas aquellas situaciones que requieran de su intervención y mantener el sistema de monitoreo y evaluación actualizado (OPP/UCC, 2014 b: 2).

\subsection{Elementos comunes a los programas de proximidad y lo que ellos muestran}

Más allá de las diferencias entre los programas de proximidad, es posible identificar elementos comunes los que serán presentados en este apartado poniendo particular foco en aquellos a partir de los cuales es visualizable la manera en que la política pública opera en la actualidad.

Como puede observarse en la documentación analizada y en las entrevistas realizadas, los programas de proximidad se dirigen a sectores particularmente vulnerables de la sociedad, lo que se enmarca en las preocupaciones señaladas por la Reforma Social. Indica un operador:

Estamos trabajando con familias desde un proyecto focalizado, donde son familias que están en una situación de vulneración de derechos re compleja en muchos aspectos, en distintos niveles de opresión que tienen (Entrevista a operador Cercanías, 2016).

Esta realidad instala en el comienzo una tensión. El trabajo con estos sectores se configura como un dato de partida, sin ser problematizado. No hay una reflexión sobre por qué la política asistencial y más aún su ampliación y diversificación continuaría siendo necesaria fuera de contextos de emergencia y con aumento del gasto social.

El acceso a bienes y servicios públicos no es solamente relevante como parte de la protección social, también lo es más específicamente en tanto los programas de proximidad se proponen vincular o re vincular a los destinatarios con el mundo formal del trabajo y el resto de la oferta pública. Pero las condiciones y características de estos tampoco son problematizadas. Parecería suponerse que existe un mercado laboral con capacidad de incorporar a todos en condiciones decentes y políticas públicas en suficiente cantidad y calidad. No hay una problematización sobre qué es lo que ha hecho que si estas existen, exista también población que no asiste o que abandona. Esto se configura como una nueva tensión, en tanto alcanzar los objetivos de los programas, se vuelve extremadamente dificil. Esto aparece expresado de manera consistente por parte de los entrevistados. 
Se busca restituir derechos, es como el marco amplio a lo que apunta la estrategia, hay una cosa que es interesante que se plantea, que es la idea "puente". El dispositivo que viene de una politica focal, como puente hacia lo universal. Asi está pensado, en la realidad no se genera ese efecto, no alcanza sólo con movimientos que se puedan trabajar con la familia. Falta la otra parte, qué hay del otro lado del puente, desde la política universal, para recibir a estas familias. (Entrevista a operador Cercanías, 2016)

Los recursos son pocos, por ejemplo el tema de la vivienda presenta muchas dificultades, así como el empleo. No le puedo seguir diciendo a la familia que se tiene que independizar y empoderar, porque los adultos no tienen mercado laboral. No tienen primaria terminada, ninguna experiencia laboral formal. No hay un mercado laboral que los absorba. Terminamos eligiendo no trabajar esos temas con la familia, porque nos sentimos que les estamos mintiendo, no le podemos dar alternativas. Los recursos no están para todos, no es solo el recurso monetario, no es que le aumentes el costo de la TUS [Tarjeta Uruguay Social], sino que haya posibilidades de trabajo. (Entrevista a operador Cercanías, 2016).

A las tensiones referidas se suma una siguiente derivada del hecho que el trabajo con familias en extrema pobreza, es realizado desde dispositivos que tienen plazos acotados de actuación y que poseen muy baja materialidad e institucionalidad. Esto significa muy poca capacidad de dar respuesta a las necesidades extremas de la población destinataria.

Es una estrategia súper ambiciosa, para el dispositivo, y para los tiempos, $y$ para todo lo que pretende. Se trabaja con familias muy pobres, que vienen en condiciones de materialidad muy crudas, es dificil poder trabajar otras cosas cuando la materialidad es tan precaria.

(Entrevista a operador Cercanías, 2016).

De acuerdo a los entrevistados, un aspecto clave del trabajo de proximidad refiere al "ir hacia", tomar contacto con población que está por fuera del entramado institucional y llegar a una población que el resto de los programas no llega. En este sentido señala un coordinador:

Los dispositivos de proximidad, son aquellos que trabajan con familias que por distintas razones no acceden a los servicios por sí mismas. O que han tenido un tránsito por distintos servicios de los dispositivos tradicionales, donde no han tenido respuestas, $y$ por tanto necesitan de un acompañamiento cuerpo a cuerpo. Estos dispositivos vienen a llenar esos vacíos para lograr que esas familias accedan a prestaciones $y$ a la restitución de sus derechos.

(Entrevista a coordinador Cercanías, 2016).

Este "ir hacia" la población destinataria de los programas, se da en un país donde la presencia pública hace que prácticamente toda la población haya tenido contacto con las políticas sociales en alguna de sus expresiones. Pero más aún, en muchos casos la población de los programas de proximidad es derivada por otros programas, incluso por aquellos a los cuales se espera que vuelvan una vez finalizado el tránsito por los programas de proximidad. De esta manera, estos muestran a sujetos que han tenido vínculos con el Estado. Escuelas, centros de salud, servicios de atención a la primera infancia, forman parte de los actores que derivan a los programas de proximidad. Señala un operador:

En general trabajamos con familias que son derivadas ya sea de centros de salud o de algún otro agente especial, que detecta casos, que podría estar dentro de los requerimientos del programa.

(Entrevista a operador UCC, 2016).

En algunos casos, los entrevistados refieren a esas trayectorias -fracasadas- de vinculación con las políticas sociales.

Lo que fundamenta esta estrategia para estos jóvenes es una trayectoria de desvinculación previa, en la cual las demás instituciones no han llegado a ser referentes significativos, ni de lo educativo ni de lo laboral, pero también de otros componentes sociales, los cuales están marcados por la exclusión y las demás instituciones no llegan fácilmente, $\gamma$ ahí creo que la estrategia de proximidad pasa a ser la metodología que eligen para llegar a estos gurises. (Entrevista a supervisor de Jóvenes en Red, 2016).

Se coordina mucho con los servicios de la zona, sobre todo de salud. Cuando hay familias que tienen dudas sobre el cuidado de salud de sus niños, si dejaron de ir a controles, ahi se coordina para que vayan. Se busca que puedan volver a vincularse, encontrase con su historia clínica otra vez, que se 
pueda determinar por parte de los profesionales de la salud qué es lo que hacer.

Entrevista a operador UCC, 2016). no funciona, porque no funciona lo interinstitucional $y$ en realidad no hay respuesta distinta a nada.

Entrevista operador Cercanías, 2016).

En otros casos se trata de una institucionalidad que está literalmente ausente. Así, otra tensión se deriva de la puesta en marcha de programas que pretenden vincular a una institucionalidad que no existe, tal y como surge de las entrevistas realizadas:

vios, muestran un Estado que termina contribuyendo a "la necesidad del trabajo de proximidad", lo que tampoco aparece problematizado, sino configurado como dato. En tanto no se construye una explicación del camino que lleva a esta situación, tampoco se elabora una respuesta.

Estos programas permiten ver las formas de actuación del Estado y en particular sus políticas sociales. Más allá de la orientación que las mismas tengan, terminan teniendo una práctica que excluye a distintos sectores sociales. Así, se dice en una de las entrevistas:

Los gurises arman su puzle de acuerdo a lo que reciben $y$ lo que reciben es que el Estado falló.

(Entrevista a operador Jóvenes en Red, 2016).

Los objetivos de los programas de proximidad se alcanzarían a partir de diseños interinstitucionales y del trabajo de proximidad. En relación a lo primero, es posible señalar también algunas tensiones.

La interinstitucionalidad -señalada como parte de las innovaciones de los programas- se configura como un presente-ausente. No aparece efectivamente y es posible ver que el Estado vuelve a estar omiso. Es contundente el relato de los entrevistados en relación a las dificultades para viabilizar nuevos y diferentes recursos. Los programas de proximidad muestran una institucionalidad pública que protege de forma desigual e insuficiente a los ciudadanos

Cuando se plantea la estrategia, aparece una cuestión de interinstitucionalidad que se supone que funciona. Hay un montón de cosas que tienen que ver con esos protocolos que no son reales. Por ejemplo, para conseguir un cupo para un refugio, pasamos por lo mismo que cualquier otro programa. Con vivienda, hay un protocolo de mitioación que es el mismo que para todo el mundo, y que son prestaciones que no se adecuan a las familias. No puede ser terreno inundable, no puede ser rural, no puede ser privado. La gran mayoría queda afuera, pero si encontrás un caso en que sí, y presentás ese formulario, pasas un par de años, $y$ no hay resolución. Hay algo de los protocolos que
Trabajamos con gurises que terminaron sexto de escuela $y$ tienen 21,22 años y desde que dejaron la escuela a los 12 no se anotaron en nada. Cuando empecé a verlo me parecía increíble pero claro en este barrio no hay un centro juvenil y no había liceos, la escuela tenía primero, segundo y tercero en modalidad rural, recién el año pasado se inauguró la UTU [Universidad del Trabajo del Uruguay] y está hasta el tope y los gurises con lista de espera porque está eso del boleto. Arrancás las clases en marzo, hay que pagarse el boleto para ir a clases porque las boleteras aun no están activadas, ahí si tu familia no tiene ni para comer $y$ te quedaste sin lugar en la UTU del barrio no vas poder estudiar.

(Entrevista a operador Jóvenes en Red, 2016).

Yo digo que somos puentes, que el problema a veces es justamente ese, que nosotros tratamos de conectar pero del otro lado no hay respuesta. Con CAIF [Centros de Atención a la Infancia y la Familia], nos pasa que tratamos de hacer una coordinación pero hay lugares en los que no hay ninguno alrededor, tenés uno a dos kilómetros o más. Lo mismo con las policlinicas y las instituciones educativas, tratás de motivar a las mujeres para que hagan un curso y salgan de sus casas pero no tenés nada para ofrecerles.

(Entrevista supervisor UCC, 2016).

O señalan los entrevistados, una interinstitucionalidad que no avanza más allá de los acuerdos entre las autoridades:

Coordinar es un problema, articular es un problema...nosotros hemos tenido muy buenas experiencias con algunas instituciones y con otras estamos en cero, porque cambia la gente o hay visiones diametralmente opuestas y lograr pun- 
tos de contacto es tremendamente dificil. También sucede que se hacen acuerdos a nivel de cúpula y después la bajada es sumamente dificil, excesivamente compleja.

(Entrevista a operador Jóvenes en Red, 2016).

No se trata solamente de una institucionalidad escasamente presente y por tanto con muy baja capacidad de responder a las necesidades de la población, sino que en algunos casos es una institucionalidad atrapada en las perversas lógicas de la focalización:

Si vos diseñás un dispositivo en el cual tu objetivo es la restitución de derechos, no te puede quedar gente por fuera del acceso a determinadas prestaciones. Tienen que seguir trabajando mucho más eso, incluso algunas cosas que a veces aparecen, cuando la persona accede a un empleo, que le caen ciertas prestaciones. A veces es casi un cambio de figuritas. (Entrevista a coordinador Cercanías, 2016).

Las tensiones visualizadas se vuelven más dramáticas en tanto se está ante programas que implicarán un aumento de la demanda, sin que existan los recursos para responder a la misma. En la documentación analizada y cuando aparece en las entrevistas, la referencia a la institucionalidad pública está centrada en aspectos como la coordinación y la fragmentación. La persistencia de la pobreza y la desigualdad parecerían pasibles de ser atendidas desde un cambio en las formas de gestión. Parece suponerse la existencia de servicios que tanto en cantidad como en capacidad y calidad de atención podrán incorporar a sus destinatarios, lo que no se da en la realidad.

Así, las ideas de "puente" y del "otro lado del puente" pese al reconocimiento de su relevancia, tienden a aparecer como un dato y corren paralelas al reconocimiento de la desprotección del Estado. Esta realidad no parece formar parte de un debate que trascienda el relato de quienes operan en los programas de proximidad.

Uno se queda muy corto cuando necesita otros recursos, (...) en temas de violencia doméstica, nosotros llegamos a detectar situaciones graves y te preguntás, ¿qué hacés? Terminan rebotando en todos los servicios porque no dan abasto. Vos movilizás un montón de cosas que después no tenés recursos para dar respuesta a eso.

(Entrevista a operador UCC, 2016)

Estoy trabajando con una madre lo que tiene que ver con salir a trabajar, empezamos a trabajar todo lo educativo previo, la importancia de la salud, vamos al odontólogo, vamos a que te hagas el Papanicolau, hacemos el carné de salud, hacemos un curriculum. Perfecto. Pero por ejemplo si la familia tiene niños y decimos vamos a vincularlos a un CAIF, no hay cupo. Y pasa un mes, dos meses y después la familia dice 'me salio un trabajo, pero no pude porque no tengo CAIF y no hay nada en la zona que me lo pueda cubrir. Ahí es donde el equipo empieza a remarla para que la familia no desista. Porque cuesta trabajar, todo eso costó. Primero generar el interés en la familia, que diga sí, probemos algo nuevo. Se engancha con la idea que le llevamos y empezamos a trabajar todo lo otro y mirás para atrás y no está lo que necesitas. Que es la pata complementaria importante para sostener la propuesta que trabajaste con la familia.

(Entrevista a operador Cercanías, 2016).

Se trata de una institucionalidad que no parece estar problematizada por lo que los programas de proximidad expresan. De acuerdo a algunos entrevistados, a partir de estos programas existe la posibilidad de una llamada telefónica, pero parece estarse dando poca cosa más.

Si pensamos si son las políticas sociales de este tipo las que van a sacar a la gente de la pobreza, y no. Y si esto tiene incidencia en la gestión de las políticas sociales, creo que está teniendo muy poco. Ciertamente los referentes de las distintas instituciones atienden el teléfono frente a demandas, pero se hace lo que se puede con lo que se tiene. (Entrevista a supervisor Cercanías, 2016).

El otro componente desde el cual se alcanzarían los objetivos es el trabajo de proximidad, el que es visualizado como una innovación. En tanto la institucionalidad es un presente - ausente e instalado como dato, es posible observar que es el trabajo de proximidad con los destinatarios lo que adquiere centralidad en la práctica cotidiana. Esto es visualizable tanto en la documentación analizada (especialmente la especificación de tareas de los operadores) como en el relato que realizan los entrevistados sobre su trabajo. Por momentos, la prestación a ofrecer parece ser casi exclusivamente el operador, el vínculo y la escucha.

El trabajo de proximidad básicamente tiene que ver con un cuerpo a cuerpo, con un acompañamiento, con un tiempo compartido y empezar a acordar objetivos comunes, plazos, metas y estrategias para llegar a eso. (Entrevista a supervisor Jóvenes en Red, 2016). 
Entiendo que la política fue pensada para que estas familias estén priorizadas al momento de acceder a prestaciones, servicios, a lo material. En territorio muchas veces eso no pasa y muchas veces lo que pasa es que el equipo queda más como acompañando, generando espacios de escucha, de problematización y me parece que la falla está ahí, porque estas familias necesitan de lo material para poder organizar más lo interno.

(Entrevista a operador de Cercanías, 2016).

El trabajo de proximidad es entendido por la totalidad de los entrevistados como necesario y también poseedor de características singulares. Además de ir hacia una población excluida, habilitaría un tipo de intervención que se la entiende original y particular y que no existiría en el resto de la oferta pública:

La estrategia de proximidad es el trabajo cara a cara con la familia. Tenés otra cercanía, que no conseguís en un consultorio. Es otro tiempo que tenés con las familias y se genera otro conocimiento de la situación y te permite ese vínculo estrecho de confianza que es lo que habilita la intervención. Es la estrategia que está dando más resultado en el sentido de que dada la gran vulnerabilidad que tiene la población con la que trabajamos, implica que otras estrategias no permitan abordar a fondo la problemática . Entrevista a operador UCC, 2016).

[El trabajo de proximidad] me parece pertinente $y$ estoy convencida que hay adolescentes y jóvenes, seguramente en el resto de las edades tambien, con los cuales la estrategia de proximidad y el establecimiento de una relación socioeducativa, generar un referente cercano, en muchas situaciones es el único enganche posible. Para mucho es quizás la única vía de volver a transitar por espacios de inserción después de estar excluidos completamente, estamos hablando de volver a la policlínica, a la escuela, al liceo, $y$ reconstruir ese camino para los gurises solos es muy dificil. En la medida que esta referencia pueda generar un vínculo con el cual eso se transite, lo hace viable, en otras situaciones creo que no.

(Entrevista a supervisor de Jóvenes en Red, 2016).
En el trabajo de proximidad y en lo que hace al interés de este trabajo, es posible también visualizar algunas tensiones y paradojas.

La derivación de parte de los programas implica o bien (y sin más) desresponsabilizarse de algunos sectores sociales y/o entender la necesidad de un trabajo como el que es realizado por los programas de proximidad, lo que surge en el relato de algunos entrevistados.

A veces vienen de una escuela que entiende que se necesito un equipo de proximidad como el nuestro. (Entrevista a operador Cercanías, 2016).

Puede ser que un Centro CAIF visualice una situación que entiende que es necesario un acompañamiento más individual. (Entrevista a operador UCC, 2016).

En cualquiera de los casos, es posible ver -nuevamente- ausencias del resto de la política social. Si se concluyera que algunos sectores, para acceder a servicios básicos (educación, salud, prestaciones) precisan un acompañamiento particular, resulta dificil de explicar por qué no se han desarrollado mecanismos que lo hagan posible desde la oferta pública existente. La realidad atendida desde los programas de proximidad, se resuelve vía derivación a estos y no de modificaciones de las prácticas de los programas sociales, incluso en algunos casos, se señala que estos "descansan":

Lo de cercanías es porque esas familias con las que se trabaja, necesitan un tipo de acompañamiento cuerpo a cuerpo, debido a que estamos hablando de familias en situación de extrema vulnerabilidad social. Es como que están hundidas en un pozo y necesitamos sacarlas para que después puedan moverse solos. Esta proximidad lo que permite también es descansar a los otros equipos, las otras instituciones que estuvieron trabajando con la familia y no pudieron o están desbordados o se les terminaron las estrategias para el abordaje. Entonces lo que permite cercanías es eso, conocer al otro, escuchar la necesidad del otro para poder fortalecerlos. $Y$ a su vez, trabajar en conjunto con las instituciones que ya están, porque después son ellos los que quedan. (Entrevista a operador Cercanías, 2016)

Lo señalado no supone demonizar la institucionalidad pública, pero sí reconocer que muchas veces sus dinámicas de funcionamiento son efectivamente excluyentes. Vale 
preguntarse si los programas de proximidad no terminan contribuyendo a despresponsabilizar al resto de la oferta pública y a continuar consolidando una autopercepción de un trabajo realizado adecuadamente.

(Leopold et al, 2015)

Tampoco se visualiza una problematización que permita distinguir metodología de trabajo de programa. Suponiendo que esta forma de abordaje fuera necesaria, ¿es preciso también un programa particular?, ¿no es posible y pasible de ser realizado desde el resto de la oferta pública, fundamentalmente aquella a la cual se pretende vincular?.

El trabajo de proximidad además habilitaría a los destinatarios a comprender aspectos esenciales para su vida y/o acceder a prestaciones que algo reducirían la precariedad de las condiciones de vida.

Una estrategia de proximidad tiene mucha riqueza desde el punto de vista de que se puede trabajar desde la dimensión educativa promocional cosas que muchas veces desde el sistema de salud, o desde otras instituciones o programas están como dichas y no los están en realidad. A las embarazadas se les indican Papanicolau, examen de sangre, exudados, como si las mujeres todas manejaran lo que es un Papanicolau, lo que es un exudado, muchas veces hay mucho mito sobre eso. Entonces poder tener la posibilidad de cercanía, de poder ir trabajando esas cosas con las familias, ir trabajando que la indicación del hierro no es solamente un capricho médico, sino que tiene todo un fundamento detrás en la prevención de la anemia. Cada pequeña cosa de esas trabajada a nivel del hogar y al nivel de una dimensión educativa promocional creo que es muy valiosa. (Entrevista a operador UCC, 2016).

Otro aspecto es el acompañamiento a las familias, porque son excluidas de los servicios. La policlínica está pero no va, el caso más paradigmático es la AFAM [Asignaciones Familiares] en el BPS [Banco de Previsión Social]. S mandas a las familias solas a tramitar esto en el BPS, entran y salen sin la AFAM a la que tiene derecho, $y$ sin saber por qué no se la dieron. Cuando viene el "primer rebote" en el mostrador, ahí está el operador con la familia, que pide explicaciones, y ahí el trámite se consigue.

(Entrevista a supervisor Cercanías, 2016).
Todos destacamos mucho lo que es el vínculo con las familias, ellos también te lo devuelven cuando te cuentan sobre sus experiencias con otras instituciones... fui a tal lugar y me trataron horribles. Nosotros detectamos muchas cosas que se podrían evitar si alguien se tomara dos minutos para explicar una cosa, pasa con los métodos anticonceptivos, con los trámites... a veces nos toca explicar esas cosas también. (Entrevista a operador UCC, 2016)

Así, los programas de proximidad terminan realizando acciones y actividades que claramente debieran ser hechas por el resto de las políticas sociales. Estas tienen problemas si es preciso un operador de un programa de proximidad para explicar la importancia del hierro, del PAP o para tramitar una prestación a la que tienen derecho.

El trabajo de proximidad también permitiría dar cuenta de las singularidades de los destinatarios, de aquellas que se indica contribuyeron a la desvinculación y exclusión.

En teoría me parece que [la estrategia de cercanía] está bien pensada y los inconvenientes vienen luego, en la implementación. Pero en lo que refiere a la forma -de proximidadte hace conocer en detalle las particularidades de cada una de las familias. Que son esas particularidades las que las dejan por fuera. Por más que tengan cerquita a las redes, son lo que las dejan por fuera. Entonces creo que esto de la proximidad, el operador que hace ese nexo, me parece básico. (Entrevista a operador Cercanías, 2016).

Estos gurises todavía no tienen algunas estrategias necesarias para poder llegar a esas instituciones universales, que en el mejor de los casos los van a tratar a todos por igual $y$ precisan de estrategias que se adapten un poco más a sus necesidades y posibilidades. Necesitan de alguien que pueda pensar una estrategia para él o ella. (...) Sabiendo que esta amortiguación social se va a terminar y luego van a tener que adaptarse, sea en el liceo o en un trabajo, este acompañamiento tiene que servir para que se integren plenamente, no pseudo integrarse.

(Entrevista a supervisor Jóvenes en Red, 2016)

Tampoco aparece problematizada la secuencia que permitiría que la relación destinatarios de los programas de proximidad - políticas sociales que no funcionó, lo haga 
sin que se procese ningún cambio en la cantidad y calidad de la oferta pública. No queda claro cómo se podrá realizar el tránsito desde ese dispositivo focal y tan singular a espacios que una y otra vez se los entiende como hostiles y los cuales -como fuera indicado- se toman como datos.

[El trabajo de proximidad] es fundamental, no hay otra forma de trabajo. Si esperás que desde las instituciones formales te manden algún tipo de información o sigan a los gurises más de cerca, es imposible. Nosotros nos matamos tratando de localizar a algún profesor, adscripto o director que sea buena onda y que nos ayude a seguir con el trabajo...y es muy dificil. La cabeza del sistema formal es muy estructurada, nosotros tenemos gurise que los mandaban para atrás porque no tenían uniforme. Es una cabeza poco democrática, a la educación formal a veces le falta ese lado humano y es en definitiva lo que los expulsa. (Entrevista a coordinador Jóvenes en Red, 2016).

Parecería que los programas de proximidad quedan atrapados en su discurso de la excepcionalidad. Es una forma de abordaje tan particular, que parece ser solamente realizable desde allí.

A partir de lo señalado, se observa en las entrevistas una confianza que por momentos parece desmedida en el trabajo posible a partir de la palabra y el acompañamiento. Parecería que este habilitaría a los sujetos a transitar por los lugares donde no fueron o no permanecieron.

Lo que se busca es fortalecer a las familias, acercarlas a los servicios y prestaciones que están a disposición. Con mejor o peor calidad, eso se puede discutir. Pero me parece que busca eso, fortalecer a las familias.

(Entrevista a operador Cercanías, 2016).

Mucha gente no podía acceder a diferentes servicios del Estado, a instituciones, no se le reconocían sus derechos. Se logran muchas cosas a partir de este tipo de estrategias. Los servicios están muy recargados, no alcanzan los recursos, no funciona todo como tiene que funcionar. (Entrevista a operador UCC, 2016)

Este tipo de dispositivo habilita acercarte de otra manera a una población que sabes que tiene un conjunto de vulnerabilidades y en definitiva poder trabajar para la mejora de sus posibilidades desde otro ángulo y terminar acercándolos a esos servicios a los que no estaban accediendo. (Entrevista a operador UCC, 2016).

En algunos casos los entrevistados visualizan límites en el trabajo:

Todo ese trabajo que uno hace en la trama más subjetiva, si no tiene una contraparte de las sectoriales del Estado que den una respuesta a la integración...no camina. El guri tiene que tener la posibilidad de integrarse efectivamente, de poder trabajar, estudiar y que esa institución no lo vuelva a echar. La integración no depende solo de lo que nosotro. hagamos con los sujetos excluidos, si pensamos eso una vez que lleguen los van a terminar rebotando igual. Tanto el trabajo como la educación siguen siendo sumamente expulsivos para este tipo de población. Eso es la clave para una política social de proximidad, que esté el compromiso interinstitucional, que no sea el recurso humano puesto en juego para que el tipo se vea más fortalecido sino que además haya un Estado que pueda integrarlo.

(Entrevista a supervisor Jóvenes en Red, 2016).

En todo caso y más allá de diferencias entre los entrevistados, esa confianza en las posibilidades de la proximidad encuentra límites en la propia realidad, teniendo como otra de sus expresiones el egreso de los programas. Este muestra los límites de la palabra y el acompañamiento y vuelve a poner en tensión al resto de la oferta pública. Una parte importante de los egresos ocurren por la finalización del plazo estipulado y no por el cumplimiento de las metas establecidas y se espera que las familias queden con referencia en alguna institución.

Siempre la idea es que el niño pueda quedar vinculado con algún centro de atención a la Primera Infancia, algún CAIF, algún centro de Aldeas, para que el equipo de ese centro quede como referencia para continuar alguna gestión que haya quedado pendiente o para que la familia quede con el respaldo de un equipo técnico. Muchas veces eso no pasa, pero se intenta en la medida de lo posible que el niño pueda quedar vinculado a alguno de estos centros. (Entrevista operador UCC, 2016).

Esta realidad, en muchas oportunidades genera problemas entre los servicios, lo que podría comprometer posteriores coordinaciones. Señala un entrevistado: 
Los egresos están muy mal pensados, nos desbordan a todos los servicios, pero el protocolo nos obliga a estar en esa situación. Entonces los equipos de territorio derivan a otros equipos para el egreso, $y$ los desbordan y hay fricciones por eso. (Entrevista a supervisor Cercanías, 2016)

Después del programa de proximidad, la realidad parece ser la misma existente al ingresar.

Nosotros cuestionábamos que la familia egresa y que no hay nada para ofrecerle, que se mienta en esta cuestión de traspasos que no son reales. Las familias vuelven a los lugares donde fueron derivadas, así que nosotros al año y medio le estamos devolviendo a las familias. Eso hace ruido. Tampoco es que la familia pasa por el año y medio de la estrategia y mejora sustancialmente. Ahora la consigna es elijan las familias que les parezca importante que tengan un seguimiento, entonces eso es más real. La realidad es que hay un montón de familias, que no tienen traspaso.

(Entrevista a operador Cercanías, 2016).

\section{Síntesis y conclusiones}

Este trabajo parte de observar que en América Latina, se ha venido desarrollando un proceso de ampliación, diversificación e institucionalización de la política asistencial. Esta tiene como expresión fundamental los programas de transferencias condicionadas, sumando luego programas de acompañamiento.

Este ha sido el camino seguido también en el Uruguay a partir del año 2005, cuando se crea el Ministerio de Desarrollo Social y se ponen en marcha el Plan Nacional de Atención a la Emergencia Social y luego el Plan de Equidad.Ya en el segundo gobierno del Frente Amplio, se aprueba la Reforma Social y en ese marco se implementan los programas de proximidad que constituyeron el objeto de análisis de este trabajo.

Estos programas son pasibles de ser analizados desde diversos ángulos. Aquí el foco estuvo puesto en visualizar y problematizar aquellos aspectos que la existencia de esto programas muestra sobre el resto de las políticas sociales y esto como expresión de la desigualdad social. El análisis permite visualizar tensiones, algunas propias de los programas de proximidad y otras del resto de la institucionalidad pública.

Los programas de proximidad se dirigen a sectores que se encuentran en condiciones de extrema vulnerabilidad. Claramente no es posible pensar en atender esta realidad desde una única acción y menos aún, desde la política asistencial. Pero más allá de esto, ponen en marcha dispositivos que tienen plazos acotados de actuación y muy baja materialidad y por tanto, muy poca capacidad de atender algunas de las enormes necesidades que tienen los destinatarios.

Una de las apuestas es a la interinstitucionalidad, lo que implicaría respuestas de parte del resto de las instituciones públicas, lo que no parece verificarse en la realidad. En algunos casos los programas se llevan adelante donde el entramado institucional es casi inexistente y donde está presente opera de forma básicamente ausente. También se observan dificultades para que los acuerdos entre autoridades se expresen en los territorios particulares y en cambio priman los vínculos personales. Todo esto aparece escasamente problematizado. Las dificultades para el acceso a la provisión pública se configuran como dato sin lograr generar un debate sobre esas ausencias, interpelando por esto al Estado.

Tampoco es observable una problematización sobre la calidad de la oferta pública. No ingresa en la discusión el hecho de que una parte importante de los destinatarios de los programas de proximidad muestran una trayectoria de vinculación previa con las políticas sociales. Se trataría de llegar a una población a la que estas no llegan, pero que en su mayoría, sí había tenido contacto con las políticas sociales. Incluso son derivados desde políticas e instituciones (policlínicas, clubes de niños, Escuelas, Centros CAIF) a las cuales se espera que los destinatarios vuelvan.

El trabajo de proximidad está justificado en tanto habilitaría un tipo de abordaje que no sería posible en el resto de las políticas públicas: falta de tiempo, de disposición a explicaciones de aspectos básicos, relaciones jerárquicas y distantes, tratamientos homogéneos son parte de los problemas que los entrevistados identifican. Esto tampoco aparece problematizado y queda sin estar claro como se hará el tránsito a los dispositivos universales o porque no se discute sobre las formas de atención de estos.

En algunos casos a partir de la constatación de la institucionalidad ausente y en otros derivado de una confianza que parece desmedida en las posibilidades del trabajo de proximidad, el resultado termina siendo que es este trabajo con los destinatarios el que básicamente ocupa la práctica de lo que los programas hacen. No implica que se desconozca la relevancia del acceso a bienes y servicios, pero no forma parte de lo que los programa de proximidad efectivamente realizan. No hay acciones orientadas a que la oferta pública modifique la cantidad y calidad de las prestaciones brindadas. Cuando las políticas públicas aparecen en el discurso, lo hacen fundamentalmente asociadas a la coordinación y superación de la fragmentación. A la falta de acceso a las instituciones y prestaciones -sin que quede claro de qué manera- se pretende dar respuesta desde la proximidad. 
Pero esta orientación al trabajo con los destinatarios exclusivamente, encuentra límites en la realidad y se expresa en frustración o en un discurso que tiende a estar centrado en los micro cambios y trayectos. No quiere decir que estos no sean valorables, pero el énfasis en los micro procesos tiende a aparecer disociado de la problematización por los objetivos no alcanzados.

El rescate de la dimensión afectiva generalmente convive con el discurso de las limitaciones de las condiciones de vida, pero aparecen como asuntos paralelos y no implicados entre sí. Así, no queda claro como podrán procesarse cambios solamente desde ese trabajo, sin que nada de las condiciones materiales cambie.Y suponiendo que esto fuera posible, tampoco queda claro que pasará cuando este soporte afectivo que proveen los programas de proximidad ya no esté y prácticamente nada del entramado institucional haya cambiado.

En suma, es posible ver a través de estos programas las distintas formas en que la universalidad no aparece y en que el Estado no protege, aún en un país con una historia de intervención pública. Trayectorias de desvinculación, recursos imprescindibles que no existen (y agudizado en tanto se trata de programas que activan demandas), formas más o menos sutiles de expulsión son parte de lo que los entrevistados relatan.

Es preciso problematizar lo no problematizado, sabiendo que la no configuración de preguntas, no implica una no configuración de respuestas. Si el discurso social y político no recoge las contradicciones y desigualdades persistentes en la sociedad uruguaya y, por el contrario, tiende solo a centrarse en las mejoras operadas, es muy fácil correr el foco de la responsabilidad y la solución a las familias pobres y/o hacia quienes trabajan con ellas.

Si el discurso social y político tampoco se pregunta sobre la necesidad de programa interinstitucionales, siete años después de la creación del MIDES, del Gabinete Social, del Consejo Nacional de Políticas Sociales y del Plan de Equidad, orientados a la articulación de la oferta pública, también es muy fácil correr el foco de la responsabilidad y la solución a las familias pobres y/o hacia quienes trabajan con ellas.

Conviene advertir sobre los riesgos de la responsabilización y activación en las políticas sociales indicados por Merklen (2013: 49, 73-76). Señala el autor que la exigencia de individuación, toma la forma de responsabilización. Cada cual es responsable de su suerte y su actuación, incluyendo las consecuencias de su participación en la vida social; debe ser activo con el fin de minimizar costos en la comunidad. Las políticas del individuo apuntan al individuo como tal y no a las dinámicas que afectan la integridad o la autonomía de los individuos; toman como población a los perdedores por el hecho de haber perdido, pero no se ocupan de las reglas de juego que llevan a unos a ganar y a otros a perder, generando una situación que es al menos paradójica.
Los programas de proximidad surgen en un contexto de mejora de los indicadores sociales y esta aparente paradoja aparece escasamente problematizada. Estos programas surgen también en un escenario en que de acuerdo al MIDES y OPP (2015:9) persisten situaciones sociales de alto nivel de exclusión en muchas dimensiones de la vida, que insinúan la existencia de una sociedad dual. Señalan MIDES y OPP $(2015: 50,70)$ que en materia laboral pese a las mejoras existentes, se verifica la persistencia de importantes brechas principalmente por ingresos, sexo, edad y región que repercuten en la reproducción de la desigualdad en el mercado de trabajo con trascendentales consecuencias en otros planos de la vida. En materia educativa, en la asistencia a instituciones educativas de los niños de 3 años y los mayores de 13, en los indicadores como rezago, asistencia insuficiente y abandono intermitente y en los resultados de las pruebas PISA, se observa la persistencia de la desigualdad educativa en función del nivel de ingreso de los hogares.

Los programas de proximidad tienen una impronta territorial y son en parte dependientes de los territorios para el cumplimiento de sus objetivos. El informe de MIDES y OPP (2016: 108) indica que el análisis territorial focalizado en Montevideo y el área metropolitana muestra que la relación entre vulnerabilidad social y carencias de entorno tiene un fuerte componente territorial y se concentra en las zonas de la periferia de la ciudad y su área metropolitana. Son fundamentalmente en esas zonas, donde los programas analizados se desarrollan.

Señala Danani (2009:34-36) que la mejora de las condiciones de vida no es un atributo propio de la política social en sí misma.Tampoco constituye un atributo propio la situación contraria, esto es, que las políticas sociales empeoren las condiciones de vida. Las políticas sociales pueden mejorar la vida, pero no necesariamente lo hacen. Estas son un proceso sociopolítico, institucional, económico y cultural en el que una sociedad define los sujetos, objetos y medios legítimos de satisfacción de las necesidades. Sus diversas formas configurarán perfiles diferentes. Esto hace que lo que debe vigilarse sea el signo de esas intervenciones, existiendo omisiones e intervenciones que producen desamparo. En este sentido es preciso advertir que la política pública uruguaya -pese al imaginario que tiene de sí misma- encuentra y sigue encontrando múltiples formas de alejarse de la universalidad sustantiva al no proteger a muchos de sus ciudadanos, y así contribuir a reproducir la desigualdad social. 
Se trata de una investigación realizada por Sandra Leopold, Carolina González, Laura Paulo y Ximena Baráibar, docentes e investigadoras del Departamento de Trabajo Social de la Facultad de Ciencias Sociales de a Universidad de la República. A partir del análisis de la documentación de los programas y de la realización de 60 entrevistas a operadores, supervisores, facilitadores y referentes temáticos, el estudio estuvo centrado en as estrategias de proximidad en dichos programas, con el proposito de precisar sus definiciones, supuestos y modalidades de implene

Instituto del Niño y del Adolescente del Uruguay. Es el organismo rector en materia de políticas de infancia y adolescencia.

\section{Referencias}

Amarante, Verónica; Burdín, Gabriel; Ferrando, Mery; Manconrda, Marco;

Vernengo, Adriana; Vigorito, Andrea (2009) Informe final de la evaluación de impacto del PANES. Convenio MIDES - Universidad de la República. Disponible en: Www. nides.gub.uy. Acceso: 10 octubre 2016

Andrenacci, Luciano (2009) "Notas acerca de la política asistencial en América Latina". Revista Cátedra Paralela 6: 7-30. Disponible en: www.catedraparalela.com.an Acceso: 13 agosto 2014.

Cecchini, Simone y Madariaga, Aldo (2011) Programas de Transferencias Condicionadas Balance de la experiencia rediente en América Latina y el Caribe. Santiago de Chile: CEPAL - ASDI.

Cecchini, Simone y Martínez, Rodrigo (2011) Protección social inclusiva en América Latina. Una mirada integral, un enfoque de derechos. Santiago de Chile: CEPAL - GIZ.

Consejo Nacional de Políticas Sociales (2008) Plan de Equidad.Montevideo: IMPO.

Consejo Nacional de Políticas Sociales (2011) La Reforma Social. Hacia una nueva matriz de protección social del Uruguay. Montevideo: MIDES.

Danani, Claudia (2009) "La gestión de la política social: un intento de aportar a su problematización”. En Chiara, Magdalena; Di Virgilio, Mercedes (org.) Gestión de la política social. Conceptos y herramientas. Buenos Aires: UNGS - Prometeo Libros: 25-51.

Filgueira, Fernando (2015) "Modelos de desarrollo, matriz del Estado social y herramientas de las políticas sociales latinoamericanas". En Cecchini, Simone; Filgueira, Fernando; Martínez, Rodrigo; Rossel, Cecilia (Editores): Instrumentos de protección social. Caminos latinoamericanos hacia la universalización. Santiago: CEPAL: 49-84.

Grassi, Estela (2008) "La política social, las necesidades sociales y el principio de la igualdad: reflexiones para un debate 'post - neoliberal"'. En Ponce Larrain, Juan (Organizador). Es posible pensar una nueva política social para América Latina. Quito: FLACSO - Ministerio de Cultura del Ecuador: 29-68.

Grassi, Estela (2013) "El sujeto de la política social. Obstáculos persistentes y condiciones necesarias para el ejercicio de derechos”. Revista SER Social, v. 15, n. 33: 280 - 300 
Leopold, Sandra; González, Carolina; Baráibar, Ximena; Paulo, Laura (2015) "Las trayectorias de inclusión como estrategias de integración social. El caso de los programas Cercanías y Jóvenes en Red”. En Cuadernos de Ciencias Sociales y Políticas Sociales $\mathrm{N}^{\circ}$ 2. Montevideo: MIDES - FCS: 7-52.

Jara, Patricia y Sorio, Rita (2013) Análisis de modalidades de acompañamiento familiar en programas de apoyo a poblaciones vulnerables o en situación de pobreza. BID - División de Protección Social y Salud. Disponible en: www.publications.iadb.org Acceso: 8 de mayo 2016.

Merklen, Denis (2013) “Las dinámicas contemporáneas de la individuación”. En Castel, Robert; Kessler, Gabriel; Merklen, Denis; Murard, Numa: Individuación, Precariedad, Inseguridad ¿Desinstitucionalización del presente?. Buenos Aires: Editorial Paidós: 45-86.

MIDES (s/f) Programa Jóvenes en Red. Términos de Referencia Educador/a referente del equipo socio - educativo territorial para localidades del área metropolitana. Disponible en: www.mides. gub.uy. Acceso: 2 diciembre 2016

MIDES y Oficina de Planeamiento y Presupuesto (2013) Reporte Social 2013 Montevideo: MIDES, OPP

MIDES y Oficina de Planeamiento y Presupuesto (2015) Reporte Uruguay 2015 Montevideo: MIDES, OPP

MIDES (2016): Llamado a Organizaciones de la Sociedad Civil (OSCs) y/o Cooperativas de Trabajo para presentar propuestas de trabajo a los efectos de implementar los Equipos Territoriales de Atención Familiar (ETAF) del programa Cercanías. Disponible en: www mides.gub.uy Acceso: 23 noviembre 2016.

MIDES, MEC, MTSS, MDN, MDYT, ANEP/UTU, INAU (2012a) Programa Jóvenes en Red. Propuesta de elaboración interinstitucional. Mimeo.

MIDES, INAU, ANEP, ASSE, MVOTMA (2012b) Cercanías. Estrategia Nacional de Fortalecimiento de las Capacidades Familiares. Mimeo.

Oficina de Planeamiento y Presupuesto y Uruguay Crece Contigo (2014 a) Programa Uruguay Crece Contigo. Componente Acompañamiento Familiar y Trabajo de Cercanía. Protocolo de intervención. Mimeo.

Oficina de Planeamiento y Presupuesto y Uruguay Crece Contigo (2014 b) Términos de Referencia para la contratación de Operador de Cercanía. Programa Uruguay Crece Contigo. Disponible en: www.uruguayconcursa.gub.uy. Acceso: 22 noviembre 2016.
Soldano, Daniela y Andrenacci, Luciano (2005) "Aproximación a las teorías de la política social a partir del caso Argentino”. En Andrenacci, Luciano (Compilador). Problemas de política social en la Argentina contemporánea. Buenos Aires: UNGS - Prometeo Libros: 17-79.

\section{Fuentes:}

Entrevistas realizadas a operadores, coordinadores, supervisores y facilitadores de los programas Cercanías, Jóvenes en Red y Uruguay Crece Contigo en el marco del proyecto: Leoplod et al (2016): "Estudio de los dispositivos de proximidad en las estrategias de intervención de los programas Cercanías, Jóvenes en Red y Uruguay Crece Contigo del Ministerio de Desarrollo Social'. DTS - MIDES.

Cómo citar este artículo:

Baráibar, Ximena (2017) "¿Qué ves cuando me ves?: Acerca de lo que los programas de proximidad muestran sobre las políticas sociales en el Uruguay".

Revista Perspectivas de Políticas Públicas vol. 6 № 12:333-367. 